\title{
Application of an AIS to the problem of through life health management of remotely piloted aircraft
}

\author{
Jonathan G. Pelham ${ }^{1}$, Ip-Shing Fan ${ }^{2}$, and Ian Jennions ${ }^{3}$ \\ IVHM Centre, Cranfield University, Cranfield, Bedfordshire, MK43 OAL \\ and \\ Jim McFeat ${ }^{4}$ \\ BAE Systems, Warton Aerodrome, W374B, Preston, Lancashire, PR1 IAX
}

\begin{abstract}
The operation of RPAS includes a cognitive problem for the operators to effectively maintain their situational awareness of the aircraft and predict its health state. This has a large impact on their ability to successfully identify faults and manage systems during operations. To overcome these system deficiencies an asset health management system that integrates more cognitive abilities to aid situational awareness could prove beneficial. This paper outlines an artificial immune system (AIS) approach that could meet these challenges and an experimental method within which to evaluate it.
\end{abstract}

\section{Nomenclature}

$\mathrm{n}=$ number of dimensions in a feature hyperspace

\section{Introduction}

$\mathrm{T}$ he operations of RPAS (Remotely Piloted Aircraft System) are complex and increasingly frequent. The USAF RQ-1/MQ-1 Predator fleet has surpassed 1.5 million flight hours as of 2014. The market for small RPAS aerial survey is expanding despite regulatory concerns. The increasing use of unmanned aircraft has raised the question of safety and operational risk. If RPAS are to be used more widely they must be shown to be safe for operations near people and property. There is also a move among large RPAS designers to offer aircraft with the capability for more autonomy than has previously been used. These two trends of increased autonomy and operation in new environments both require a robust understanding of the current health state of the RPA (Remotely Piloted Aircraft) and any likely change in health state. However the ability of a remote pilot to estimate aircraft health has been called into question. This can be seen from the high mishap rates of remotely piloted aircraft in comparison to aircraft with an equivalent mission[1]. It has been shown that there can be cognitive issues with the human operators not able to completely understand the aircraft state as communicated to them through their GCS (Ground Control Station)[2]. This problem is especially acute within the management of aircraft health both during a flight and when planning the future use and maintenance of the aircraft. This paper begins with an overview of remotely piloted aircraft systems in relation to asset health management and the role operational experience and information plays. This is followed in section II-B with a look into the cognitive challenges faced by RPAS and within their management. The features which would be necessary within a cognitive asset health management system are discussed in section II-C. The immune system and its relevant features are introduced in section III and the application of AIS to cognitive asset health management for an RPAS is discussed in section IV. In section V an experiment is discussed to test the AIS and its success criteria are explained. Conclusions are then finally laid out in section VI and future work in section VII.

\footnotetext{
${ }^{1}$ PhD Student, IVHM Centre, Cranfield University and AIAA Student Member.

${ }^{2}$ Senior Lecturer, Cranfield University

${ }^{3}$ Professor, IVHM , Cranfield University

${ }^{4}$ Supportability Lead Engineer, BAE Systems
} 


\section{Background}

\section{A. RPAS (Remotely Piloted Aircraft System)}

RPAS generate a large amount of data during flight[3]. Some of this may be repackaged and sent over the telemetry link to the GCS for use by the operator to assist them in understanding the state of the RPAS and making mission decisions. The remainder is used within the RPAS by its systems. Some portion of the dataset is retained on the flight data recorder for later analysis and for investigation in the event of a mishap. This gives two sources of data which can be integrated to enable analysis of operations. This much would be present within many SMS (Safety management systems) for civil aircraft $[4,5]$. The personal experience of the pilot has often been cited as an important asset in the ability of the pilot to react appropriately in emergency situations. Understanding the aircraft state is often seen as the first step to mitigating faults and failures during flight. This presents a problem within the RPAS community as several cognitive factors have been identified which place additional burden upon the pilot in their effort to understand the current state of the aircraft[2].

The use of an IVHM (Integrated Vehicle Health Management) system to give advice to the pilot regarding mitigation options may be helpful in addressing this. Asset health management of any sort relies on a deep understanding of the asset under management, its operations, degradation modes, maintenance methods, and part requirements. During the design phase of an asset these things can be difficult to predict with sufficient accuracy. This problem is especially acute when novel systems are present which have not had IVHM systems used with them in the past. RPAS of one form or another have been in existence for almost a hundred years[6] but their modern form has not been the subject of significant IVHM work. The predator RQ-1/MQ-1 has been one of the most widely used modern RPAS. It has undergone significant development since its initial use but it has attracted criticism for its reliability and design[7]. Predator has been noted to have gradually evolved over its life cycle and has improved its mishap rate by a significant amount[8]. The evolution of design solutions can be compared to biological evolution of agents within a population. This is often cited as the survival of the fittest but can perhaps be better understood in a way that is more relevant to engineering design if we think about the situation as "survival of the most informed" (quote from Osinga)[9]. The most informed agents within a population are able to adapt to their changing environment in the most beneficial way. In order to adapt RPAS to their environment we need to ensure we are well informed regarding their use, successes, and failures. This information is needed both by the operators and the manufacturers. Despite higher mishap rates than some other aircraft the occurrence frequency of individual faults is low. This hinders the development of fault diagnostic methods through the large number of potential faults and the low volume of available case data. Across all scales from DJI Phantom all the way to the Global Hawk we need to analyse flights and aircraft management to identify areas for improvement. This is especially difficult as unmanned aircraft attract reduced investment compared to manned aircraft for health management systems. In order for the resources available to do the most good we need to create a system which can evaluate the current knowledge about the aircraft state and operational experience to highlight those sub-system areas within RPAS which would benefit most from health management. The use of the FOQA(Flight Operations Quality Assurance) technique from manned aerospace with the addition of machine learning could help identify common issues and allow a more detailed picture of the true nature of RPAS mishap. The search for meaning within this data to inform our operations is a data mining operation on a large scale and matches well with the FOQA philosophy which seeks to identify improvement and contextualise anomalies to better understand the use of the aircraft. The addition of AI(Artificial Intelligence) to draw additional conclusions which could then be supported or not by operators would need to be based on the information provided before and after each mission. 


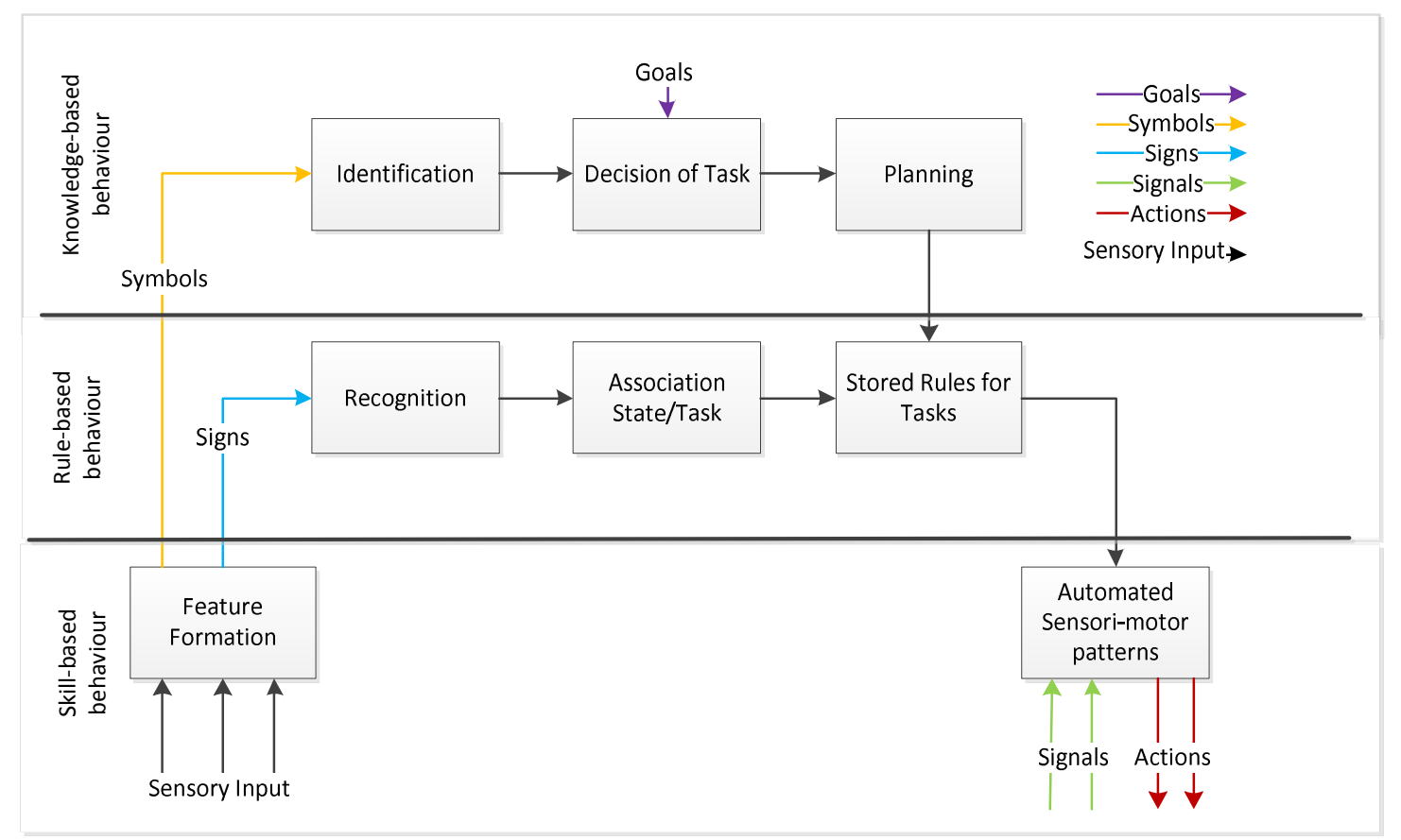

Figure 1. Rasmussen's cognitive hierarchy[10]

\section{B. Cognitive challenge}

It is worth pointing out that health information and management decisions are taken across a number of different scales of time and complexity. The cognitive concept as outlined by Rasmussen[10] (see figure 1) encounters the same stretch across timescales as Boyd's OODA (Observe Orient Observe Act) loops[9, 11].

This has particular application to the problem of health management to RPAS as there are at least four timescales of health impacting decision which may need to be made during any flight.

- Machine Decisions - Issues requiring a decision before a human could react

- Pilot Decisions - Issues which a human can decide on during the flight

- Fleet management decisions - Issues which a human can decide on before the next flight

- Strategic decisions - Issues which a corporation can decide

This is worth discussing as the system of operations and maintenance consists of multiple persons and assets with different training levels, experience, and skills. As an organisation however they must be able to actively manage their assets. Understanding their process and trying to ensure the system is formulated to work with them instead of against them is critical. Health management systems have failed on occasion due to the complexity of human-machine interaction[12,13].

In order to reduce this likelihood in future a cognitive system may offer a solution[14]. To assist in making decision in an intelligent way with a method that is transparent to the user. Despite the increasing use of more automated systems and the productivity benefits expected to be gained it seems certain that in order to gain acceptance and trust any automation must always be human centred[15]. The cognitive concept explained by Rasmussen and used elsewhere to understand the human-machine interaction includes a rule-based behaviour layer. Often explained through the metaphor

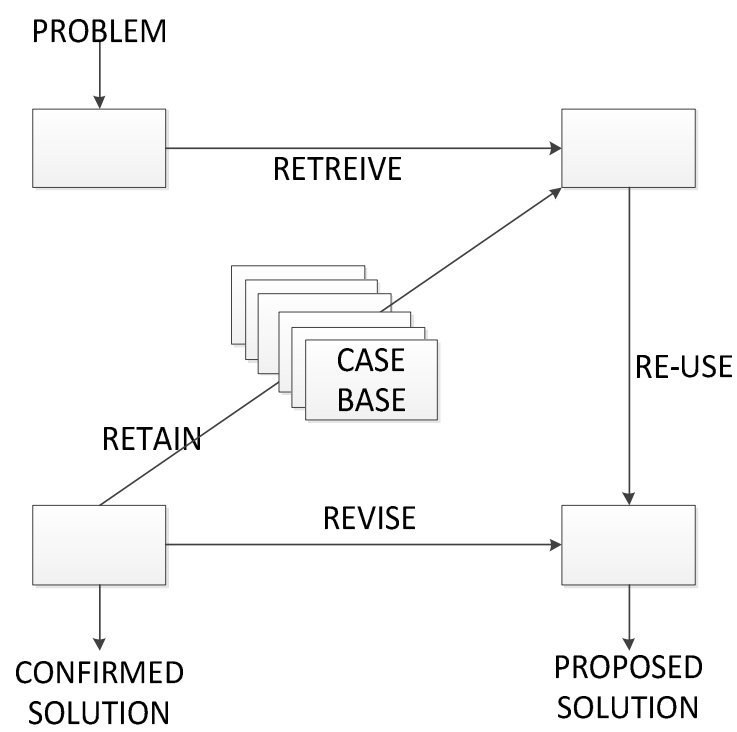

Figure 2. Case Based Reasoning[16]

American Institute of Aeronautics and Astronautics 
of single dimension control strategies this layer is also very applicable to the codification of incident response once the features exhibited by a particular incident have been recognised. The population of a set of recognition agents connected to behaviour rules is a $\mathrm{CBR}$ (Case based reasoning) structure. [16]

CBR starts from the identification of a problem and searching the case base for a similar problem. (see figure 2) The closest match is retrieved. If it is suitable it is used with its solution. If it is not suitable it is revised to make it suitable so it can be used. This new case can then be added to the case base for future use. The population of the case base for CBR can be a large problem. This makes it less suitable for the RPAS health management problem as there might be several thousand individual faults each of which may require their own case. These faults could also occur in combination to create a more complex situation which is more difficult for the operator to understand without sophisticated support. CBR is very straightforward for operators and maintainers to interrogate or add to the case base and be able to understand how they should be applied. CBR is then a good example of how a cognitive health management system might look. The use of complex systems to assist in managing the aircraft, its operations, and health involve multiple assets, resources, and humans. This can create some difficulty in creating a system which can capture issues arising and formulate responses to them as rules or schema which can then be used. Some changes may require regulatory scrutiny but the identification of rules and schema to trigger maintenance or mission actions will not. Changing unscheduled maintenance to planned maintenance at a suitable time can reduce operational disruption. This represents early maintenance rather than a situation where a maintenance credit might be needed to maintain a system later than scheduled.

Human understanding challenges

- Pilot and maintainer reports often use phrases and grammar which can be ambiguous to interpret and often does not answer questions a asset health management AI would need to know. [17]

- Pilot actions often make use of knowledge not integrated into the machine which can cause the machine to interpret input as an error.[18]

Machine understanding challenges

- Users often are forced to change their workflow to adapt to the machine rather than the machine being designed to suit the users. [19]

- Machine actions often make use of knowledge known to the pilot which can cause the pilot to interpret input as an error. Often referred to as automation surprise or cognitive mismatch.[18]

\section{Features of a more cognitive Asset Health Management system}

The system discussed here is described as a more cognitive system due to the attempt to identify the features which are needed to go a little further down the road towards a (CAHM) Cognitive Asset Heath Management system.

A fully cognitive AHM system would be very interesting but is not expected to be realized in the near future. The next steps are to go a little further using the current framework and see if this has a useful effect. This could then be built upon to further improve the ability of the system to operate in a way that is conducive to human operations managers.

(a) IVHM system within CAHM

The difference between CAHM and IVHM is that IVHM may form a part of a CAHM system but only if the IVHM system operates in such a way as to enable the whole system to be more cognitive. It is important to note that there is more to the IVHM than just health assessment(see figure 3). A more cognitive AHM will need to ensure it is has additional functionality integrated into itself. The ability to identify actions that can be taken to mitigate the fault during use is often discussed by those studying fault tolerant and self-healing methods for systems. If the fault can be successfully mitigated it can reduce the mishap rate and allow the asset to be safely recovered or at least allow the mishap to occur in an unoccupied area. It is also necessary for the IVHM functionality within the cognitive AHM

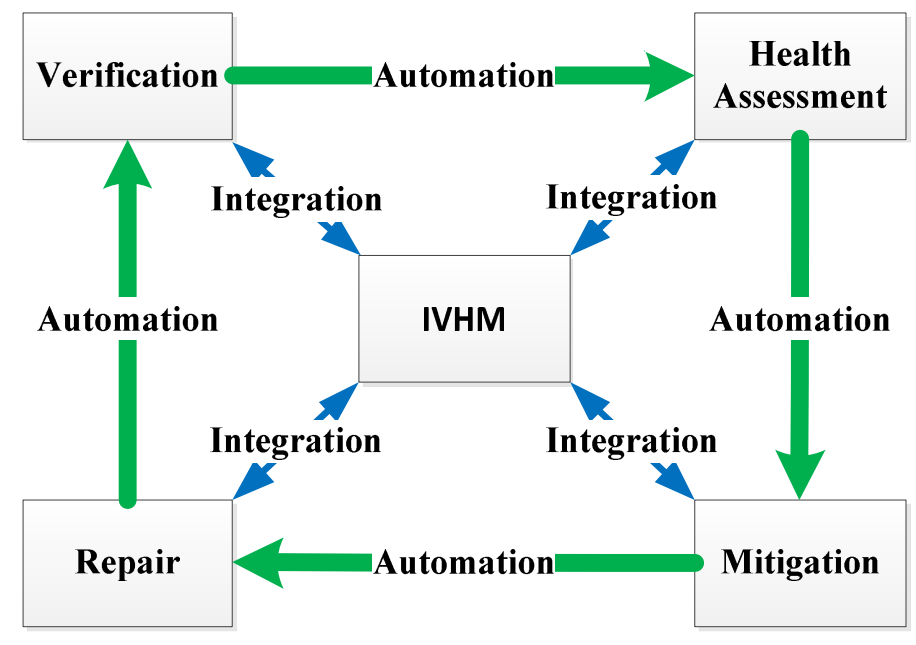

Figure 3. This is a redrawn figure 1 "Health Management Activity Model" from $[42,43]$

4

American Institute of Aeronautics and Astronautics 
to be able to assist with prediction of likely repair actions for a fault identified so that maintenance planning can be done ready for when the asset has returned. The coupling of confirmed faults with their identification criteria also improves the ability of a CAHM to identify similar but different faults. The IVHM system within the CAHM must also be able to provide some ability to verify that a repair or mitigation action has had some effect and that the effect was positive. Confirming an asset and health and ready to return to service is a key art of the IVHM/CAHM cycle.

(b) Features of a CAHM

In the features listed an RPA will be given as the example asset.

- The system will be able to be populated with a priori rules and data known to be central to the control and health management of the asset. E.g. Vne (Velocity never exceed) triggered inspection, Engine max rpm, sensor limits to trigger sensor error flag, etc

- The system will monitor the sensors and systems of the asset. Learning from use the normal behavior of the asset.

- The system will be able to identify when an operating condition has not been encountered before (novelty detection)

- The system will use machine learning to suggest rule sets appropriate to the health management of the asset

- The system will through application of rule sets to its usage history create estimates of the ROC curve of the new rules.

- The system will be able to share rules and operating information with other assets within the fleet.

- When a novel condition is identified or rule triggered the system will identify the nearest historical case in the fleet usage history to form a basis for comparison and proposed action.

- Consideration is to be given to the method by which rules and cases can be shared between assets of the same model, type, species, and genus. That is the degree to which learning can be generalized. E.g. RQ1/MQ-1 Predator Block 1 compared to RQ-1/MQ-1 Predator Block 1(same model), RQ-1/MQ-1 Predator Block 2(same type), MQ-9 Reaper(same species), and U-2S Dragon Lady(same genus)

- A method to allocate which rules (if any) to be deployed within the asset and which should be used within the system support structure instead. Aircraft have a particular need to restrict the allocation of on-board weight $\&$ power. They are however by no means unique in this.

- The system will be considerately designed to work as a native part of the operators workflow rather than an annoyance.

\section{The AIS (artificial immune system) as a candidate method for cognitive AHM}

Artificial immune systems have developed since their initial appearance in computing[20] Their original embodiment was based upon the negative selection algorithm and the self/non-self paradigm. A detector with some number of features $\mathrm{n}$ was compared using one of several available distance(affinity) measures to samples of self and if the detector alarmed it was removed from the population. This reduced the false alarm rate but is difficult to improve over time as our understanding of self and non-self would be expected to improve over time for a system which is under supervision. Data is constantly being generated during use and must be categorized into normal and potential anomalous behavior. However not all possible coordinates within the $\mathrm{n}$ dimensional feature space can be known or classified as self/non-self a priori. This creates the issue of unknown self and unknown non-self showing up as novel within the system and needing to be classified as self/non-self.[21]

The immune system exhibits properties associated with a cognitive system such as threat identification, neutralization, memory of threats, and recognition of new threats. [22]

AIS have been previously identified as a useful type of AI.

"The main features in immune systems that are very important yet lacking of today's artificial systems are robustness, adaptivity and autonomy. Therefore, these are the main functions of any artificial immune system" (Quote from Hofmeyr \& Forrest) [23, 24]

"an antigen is any molecule which can be recognized by an antigen receptor. The part of an antigen that actually fits

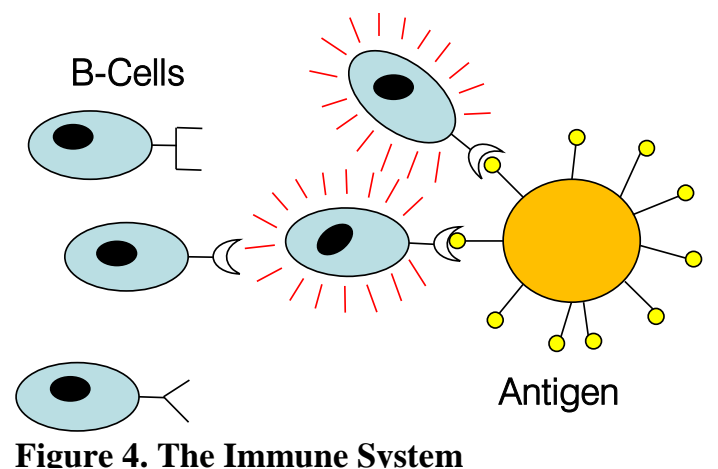


into the receptor is called the epitope of the antigen" (Quote from Cohen) [22]

Immune systems consist of a set of B-Cells which are created through pseudo random genetic variation. B-cells match themselves against the proteins found on the outsides of living cells. If it matches this causes the B-cell to bind to the antigen and release a chemical which attracts a T-Cell to come and absorb the matched cell and all the Bcells which have attached to it. This is the bodies' way of reacting to foreign bodies such as microbes. See figure 4

(a) Clonal Maturation

When a B-cell is successful in its role its production is repeated through clonal maturation. This creates replicas of the B-Cell which alarmed correctly and also creates subtlety different mutations of the B-cell which cover small differences. This allows for the system to both respond to known threats(antigens) and also respond to threats not previously identified but which share some similarity to previously identified threats.

(b) Negative selection

As the system can learn to respond to new threats and all its alert conditions cannot be mapped beforehand the immune system takes steps to ensure that any B-cells produced do not false alarm by attaching themselves to normal human health cells. It does this by a process known as negative selection. It compares the alarm patterns for each new B-cell against those patterns which have been identified as present in normal healthy cells. All those which would alarm against a healthy cell are destroyed before entering the bloodstream.

(c) Cell aging

After the cell has been created it ages and once it has reached a certain age it dies in a process called apoptosis. This helps limit the total population of cells and hence processing requirements. When a threat is detected the population is temporarily increased.

(d) Danger Theory

When a cell is under stress it can emit a danger signal which lowers the level of affinity required for a B-Cell to bind to a suspect cell. This reduces the number of false alarms in other areas where there is no stress but allows the system to deal more quickly with an issue when the antigen is not a precise match for the current B-Cell

The B-cells within the AIS have for some time formed the basis for of detection and action for AI's using this paradigm. The use of T-cells and bone marrow metaphors has not gained widespread use but may in time. The AIS is best described as a cartoon of the human immune system. It is however a cartoon with useful properties.

The identification of antigens within data depends on their correlation with some measure which has been identified as useful for classifying the end results as normal or anomalous.

\section{An AIS for through life health management of RPAS}

To manage the through life health of RPAS is a complex task which faces a number of challenges. The business case for an IVHM system is yet to be demonstrated but has been the subject of some discussion[25]. The need for an IVHM system to assist in the management of though life health has been laid out in section 0-A and the need for cognitive features within that system is discussed in section 0-B. The use within AIS of detectors modelled on Bcells creates a situation where there may be multiple cells alerting against different epitopes of the same antigen. This improves coverage of a single fault with multiple symptoms within the flight data. It also means that the occurrences of a set of symptoms for a fault can be stored as a case for future reference. The AIS shares several features with CBR and indeed AIS have been used in the past to power CBR machine learning. [26] This is useful for two reasons. One it provides an opportunity to map out the feature space around a fault. AIS use clonal maturation to create additional detectors similar to a successful detector. This covers the nearby areas within feature space in case of similar faults. This linking of historic case data to the immune cells created from it helps give context to maintainers investigating new alarms from the system. The second reason this is useful is that because the system ties case data to immune cells it acts as a data miner to create a clustered case base populated with flights where faults occurred to aid further engineering work to develop fault detection systems for the faults identified within the case base. The epitopes used by the immune cells in the case base can act as a sign to help aid identify the underlying mechanism of the fault and its degradation mode. This is because they are present within the immune cell as their presence in flight data is highly correlated with the fault for that case. 


\section{A. Representation}

Due to the large amount of real valued data the AIS should use a real value representation. These frequently scale the individual features of the schema to unit length to remove weight issues from the data. All values encountered by the AIS are therefore between 0 and 1 creating a unitary hypercube.[27]

This real valued representation takes the form of a string of data fields each with a value between 1 and 0 .

High dimensionality within AI learning can create a high degree of computational complexity and also reduce the effectiveness of some of the simpler affinity measures such as hyper spheres.[28] This creates a need to allow variable length representations within the schema so that the system can attempt to optimise for shorter schema where possible. This will also have the effect of concentrating the features used in those which are diagnostically useful rather than those which over fitting with many different features of the same fault. Coverage of multiple epitopes is better achieved through multiple cells in exchange for this reduced complexity schema.

An additional representation can be created for aircraft metadata to enable different aircraft to gain from faults identified in other aircraft. If the serial number, model number, type, and other distinguishing markers are included in a representation schema then Boolean matching can be conducted. Where the fields match a 1 is assigned else 0. This affinity measure can then be evaluated for distance through use of the hamming affinity metric.[26]

The immune cell representation will also contain such things as the creation time to enable cell aging, the current calculated ROC(Receiver Operating characteristic)[29] curve, and the case data from which the cell was created.

The case data will include the flight reference, the identified fault, the maintenance action taken, and any additional notes the operators may consider relevant.

\section{B. Affinity measures}

The use of real valued representation and bit Boolean matching means that the data portion of the schema will match using a different affinity metric to the metadata portion of the schema.

(a) Real valued representation affinity metrics

The simplest affinity metric to use is some variant of Euclidean distance calculation within the $\mathrm{n}$ dimensional feature space. This has been criticised for $n>41$ however due to the majority of the volume of the hyper sphere being located close to its surface. This reduces its effectiveness at giving a good distance measures to other locations within the space and makes it difficult to calculate its volume so an estimate can be made on the self/non-self coverage of the feature space.[28] Hyper spheres do have the benefit of being a simple distance measure however. Only a radius is needed to specify the coverage of a hyper sphere which greatly simplifies the changing of the volume of the detector coverage to adjust its ROC curve. As the dimensionality of the feature space in our simulation is less than 40 the hyper sphere method was chosen. In future other methods can be evaluated for higher dimension feature spaces.

(b) Boolean matching affinity metrics

Measuring the distance between two bit strings has been worked over several times. [26, 27] The two strings are compared using the field values and assigning a 1 for a match and a 0 otherwise. This is then evaluated for the number of matches and consecutive matches are given a higher weight due to the implied higher affinity.

\section{Maturation process}

Initial immune cells are populated stochastically to generate random string detectors. The metadata section will not be undergoing change so is not affected. The generated detectors are then presented with flight data with different settings for the radius of the matching rule. This allows an ROC curve to be built up for each detector in relation to a fault identified from previous flights. As long as the data has been classified as non-self and attached to a specific fault it can be processed. Gradually immune cells which do not exhibit enough specificity die through the aging process. New cells are generated stochastically and through clonal maturation where cells have demonstrated a high enough specificity through their ROC curve. As the cells continue to mature the lower specificity cells die off while the more effective classifiers are cloned and go through clonal maturation. This process continues until a threshold is met for the specificity of a percentage of the population.

The key is the use of some specific feature such as the early replacement of a part/LRU and binding it to a flight dataset as a classifier. The AIS can then determine what about the flight was unusual(non-self.) What differed from a normal flight. This will create a rule set which can then be used to create an advisory and also to guide future research for a more highly optimised diagnostic/prognostic method for the specific failure mode involved. Thus each time a fault occurs a case is generated for it and added to the case base. Items within the flight data are scrutinised to generate a rule set which would diagnose it. Faults sharing a common mode or subsystem should cluster. 


\section{Danger Theory}

In use the AIS can respond to stressor signals either created by individual health enabled sub-systems or in response to a danger signal initiated by the pilot. This lows the affinity requirements for alert to generate a list of the most similar immune cells with attached cases to the current aircraft condition as assessed from the flight telemetry. The case data presented can include the checklist for the fault identified within it to reduce the workload on the pilot in identifying the correct procedure to use.

\section{E. Dealing with the data}

(a) System data sources

We have listed a short summary of the type of data which we suggest would form a necessary part of a CAHM.

1) RPA in flight telemetry

This data is typically less than $10 \mathrm{~Hz}$ and has been compressed for transmission to the GCS. Only a subset of the aircrafts sensors would typically be selected for transmission during flight. Some signals may be accessible by request of the pilot however.

2) RPA QAR data (Quick Access Recorder)

This data would need to be retrieved from the aircraft between flights and can range in sample rate from 0.1 $\mathrm{Hz}$ to $10 \mathrm{~Hz}$. The number of channels varies from aircraft to aircraft. The amount of data generated during a long flight can be substantial however.[30] A simple calculation for 20 channels of data sampled at 10Hz to a 16bit ADC (Analogue to Digital Converter) for a $7 \mathrm{hr}$ flight yields $164 \mathrm{Mb}$. This is on the low end of the amount of data we should expect. RPAS have more digital control systems on board than an equivalent mission manned aircraft and are likely to log a larger amount of data. As a guide the more sophisticated civil aircraft are rumored to generate on the order of 512 Gigabytes per flight.[31]

\section{3) Maintenance reports}

During the maintenance of an aircraft reports are generated to specify what was investigated, what was replaced, and what was certified as correct.

\section{4) Flight plans}

A flight plan will typically contain the necessary information to conduct the flight. Any deviations could be the result of anomalous behavior or simply due to a change in mission tasking during the flight. It is difficult to know for certain unless mission debriefs are also available to the system in a codified way.

\section{(b) System data processing}

It is clear from the different types of data that any system to enable the through life support of RPAS and the learning through life of their faults and operations will need to be able to handle potentially very high amounts of data and also to do analysis on this data. Some of this will be highly structured such as the flight telemetry and QAR data. However some such as the flight plans and maintenance reports will not be as highly ordered. In order to reduce the cost of storing and processing data it is likely that a distributed file system will need to be used.[32] If the CAHM is structured to interrogate the system data within something like the mapReduce[33] framework this would enable parallelization and further reduction in cost for profiling the data and identifying novelty events.[34] An interesting parallel can be drawn to the world of formula 1 where they similarly make use of a distributed computing model to process the large volumes of telemetry they gather during a race in order to predict faults and identify competitive advantage.[35]

(c) Classification

Classification type machine learning or data mining has an issue regarding how the algorithm can be taught to discern which category data should belong to. Often the solution adopted is to train the algorithm on some training set and after testing to use it on the application. This assumes that the training set is sufficiently representative of the data which will be encountered in use by the algorithm. As the AIS will be expected to learn new faults during the lifespan of the aircraft a method will need to be found to classify the flight data. This method is shown in figure 4 and classifies the flight into either self or non-self. NFF(No fault found) is of concern for any diagnostic tool as the cost of maintenance action and the lost revenue from the out of service aircraft is significant. False positive diagnosis incidents will be monitored with a NFF count attached to the offending B-cell. This count would increase the rate of cell aging to remove the B-cell from the system unless. This count can also be decremented if the same B-cell has a series of successes before it goes through apoptosis. The occurrence of NFF does not necessarily mean 


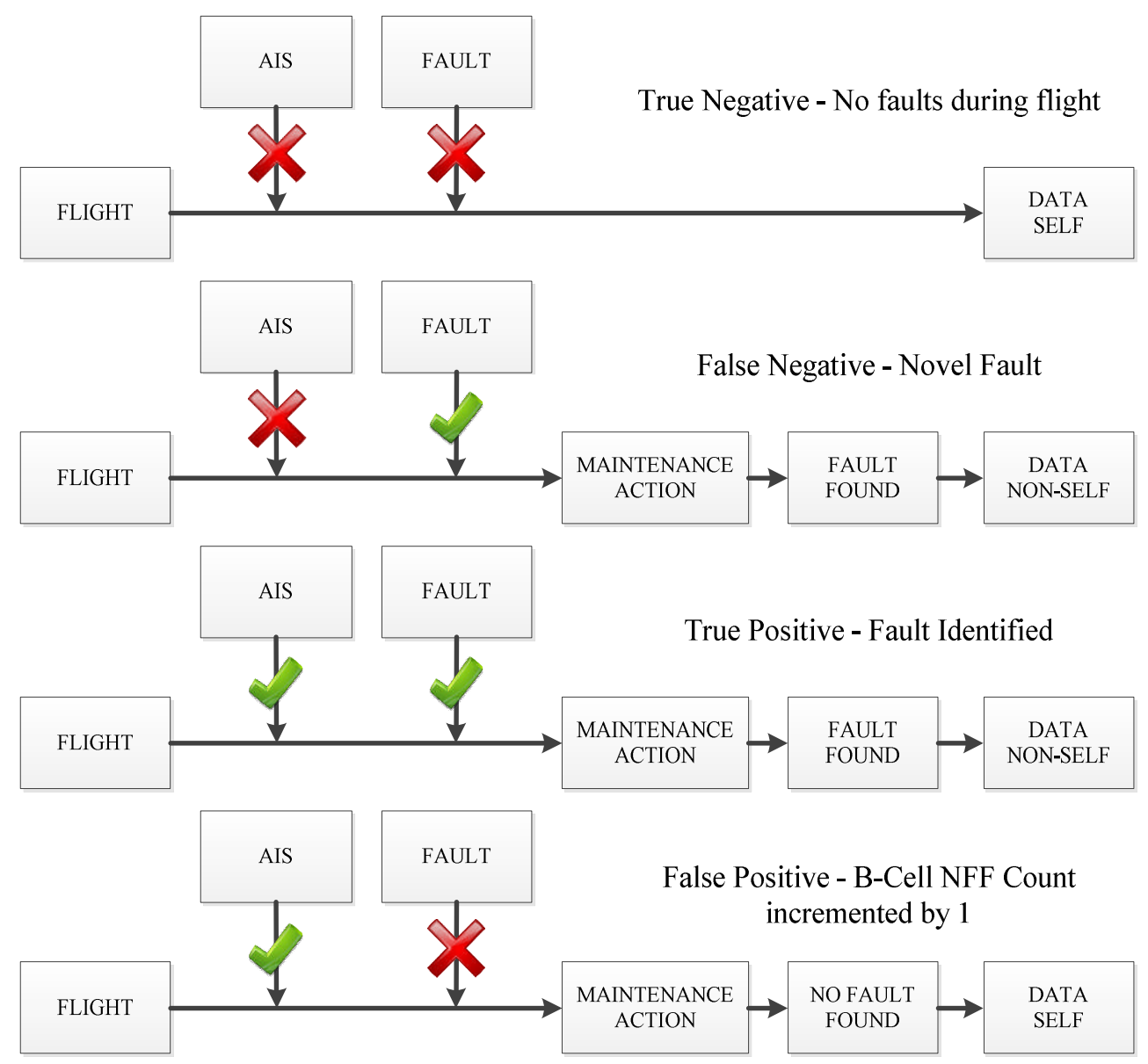

Figure 4. AIS Classification scheme

that there is no fault but it does mean that there was not sufficient information within the system to justify ignoring the alarm and also not sufficient information to identify the fault(if there was a fault which wasn't found). Careful attention to NFF and the B-cells which have NFF associated with them will be necessary. The associated cases from previous NFF from the same B-cell should at least be useful in clearing the NFF or ruling out certain faults

\section{Simulated environment and AIS evaluation}

\section{A. Experiment}

(a) Scenario

The aircraft chosen for this experiment was the Predator due to the high volume of class A mishap reports available through the USAF safety center and due to the further work done by Hartfield et al.[1,36] Two mishaps were identified for initial modelling within simulation. These were the class A mishaps of T/N 99-3058 in 2012 \& T/N 01-3075 in 2013. These mishaps were both the result of a progressive failure of the VPP (Variable Pitch Propeller) actuator servo. This was chosen due to the descriptive data in the mishap report and the nature of the failure. If the mishap pilot had identified the fault at an earlier point during the flight the aircraft could have been recovered. This represents the class of mishap where a pilot advisory CAHM/IVHM system could be of benefit. Diagnostic actions taken by the pilot due to poor aircraft understanding accelerated the failure of the servo motor. Following the initial point where the mishap pilot identified an anomaly but not the system causing the issue an alert could have seen triggered by the pilot who could have used an AIS danger signal to identify the nearest B-cells within the population and their attached fault cases. In our simulation of this scenario the aircraft will start from 50m altitude AGL (Above ground level) during the climb phase after launch. The aircraft will fly under the control of an autopilot to its cruise altitude; make its way to a point of interest and then orbit. At a preplanned point the aircraft will either move to its next tasking or return to the airfield for recover and initiate a descent for landing. The simulation will end at 50m AGL. The number of points of interest and their location will vary for each simulation to create a 
variety of paths and maneuvers within the aircraft flight data. The fault will occur at an artificially high rate of once every hundred missions

\section{(b) Simulation}

To model predator a fight model and autopilot were obtained that had been used successfully before for flight simulation research. This model and controller were of the Aerosonde RPAS [37]. This was the AeroSim Matlab block set by Unmanned Dynamics that was subsequently modified for Matlab 2014. In order to make the model behave in a way more representative of Predator the flight characteristics were altered using data found from a Predator model for an older version of Matlab[38]. The flight model and controller were subsequently modified to correctly integrate the use of a variable pitch propeller and the different weight and fuel flow rates.

A state model was then created in Matlab within which the fault was modelled. This allowed the healthy flight of the aircraft to be simulated before switching to the damage state and the damage to the servo motor gradually being increased towards failure.

A script was written to automate the generation of mission plans for the controller and model to fly in simulation so that multiple runs of health and damage including flights could be undertaken to build up the necessary training data for the AIS. This flight data during simulation is processed at $400 \mathrm{~Hz}$ but is logged at $10 \mathrm{~Hz}$ in common with other RPAS QAR and flight telemetry units in use within the university environment. This was to enable the AIS to be transferred and tested with different aircraft flight data as it became available.

(c) Fault model

Multiple state models of the aircraft are planned to allow the training of the AIS for multiple different types of fault. The limitation of this type of flight simulator is internal system faults cannot be learned due to the coarse nature of the model. [39] An aircraft manufacturer could use this technique however as they would likely possess a more detailed system architecture and dependency model created during the design and evaluation of their RPAS. The failure of the VPP servo actuator was modelled by approximating the force required to alter the pitch of the propeller at speed and then implementing a ramp input to gradually reduce the servo power until it could no longer move. This gave a fault with simple characteristics but with a subtle onset. Each mission containing a fault will end as a class A mishap within this simulation. Modelling the recovery of the aircraft departs from the limitations of the autopilot and the linear flight model of the simulation and so will not be performed within this study. It is assumed for the basis of this experiment that the flight data recorders of all crashed aircraft would be recovered and classified so that the system can learn from them.

(d) AIS Implementation

The AIS has been created with the architecture discussed earlier and processes the data for each flight to create detectors that can correctly classify the fault and the time the fault started within the mission. The algorithm is trained based on the known classification of the flight and the known initiation time of the fault. Once mature detectors have been created they are run in parallel with the simulation to diagnose faults on the fly. Each detector is updated based on their ability to classify data as self/non-self with the classification scheme shown in figure 4 .

\section{B. Evaluation}

The AIS is currently still undergoing evaluation but initial results will be presented with this paper at Scitech. The AIS is being evaluated on the basis of its ROC curve for individual faults and in comparison to other machine learning methods used for the same application such as genetic algorithms. The features which will need to be seen if the AIS is to be seen as a success are

- Good diagnostic accuracy compared to other AIS within aircraft fault identification[40]

- Good diagnostic accuracy compared to a genetic algorithm trained for the same task

- Good diagnostic accuracy compared to a back propagation neural network trained for the same task.

- Human readable alarm report stating the epitope for the B-cell

\section{Conclusions}

The through life management of RPAS is a complex topic which requires a more in depth analysis that is currently possible. The competitive nature of business and the confidentiality requirements of some funding reduce the ability of researchers to identify core faults and issues within RPAS that could be addressed. This also hampers the ability of researchers to do reproducible research to a gold standard.[41] Comparison between different AIS methods is needed for the field to really improve. The use of flight data analysis holds real potential for both identifying areas within RPAS for future investment and for identifying cognitive rules to assist in health management while we develop the next generation CAHM and IVHM systems. AIS shares useful similarities with CBR and the cognitive 
model of human machine interactions which can potentially help create a system which is more able to communicate the aircraft health state to the pilot with the necessary context for them to understand and the necessary recommendations for them to take suitable action. RPAS generate large amounts of data and as costs for storage and processing continue to fall it will be higher quality data and we will be more able to process it and learn useful lessons to inform future operations.

\section{Future work}

It is planned to add additional faults to the simulation to test the ability of the AIS to cope and how the immune population size would need to change to cope. A new flight model Predator is also planned to be introduced with slightly different characteristics to evaluate the ability of the AIS to generalize fault modes across similar bt different aircraft. The inclusion of a NFF model within the AIS classification system in order to evaluate the reaction of the system to different rates of NFF. This cannot be evaluated within the current experiment due to the class A mishap nature of each fault. The flight data of the simulation will be further partition to create a $10 \mathrm{~Hz}$ telemetry stream during the simulation for a simulated GCS within which the AIS can run. The AIS would be split to investigate which AIS immune cells are suitable to be run within the RPA where $400 \mathrm{~Hz}$ data is available but less computational power.

\section{Acknowledgments}

We would like to thank BAE Systems for their sponsorship and assistance.

We would like to thank EPSRC for funding the project.

We would also like to thank the IVHM Centre and our colleagues.

We would like to thank Dr Whidbourne for his assistance with the simulation model of Aerosonde and other RPAS.

\section{References}

References

[1] Hartfield, R., Carpenter, M., Randall, W., "Unmanned Air Vehicles (UAV): Safety Event Prediction, and Classification," 12th AIAA Aviation Technology, Integration, and Operations (ATIO) Conference and 14th AIAA/ISSMO Multidisciplinary Analysis and Optimization Conference, American Institute of Aeronautics and Astronautics, 2012,

[2] Neville, K., Blickensderfer, B., Archer, J., "A Cognitive Work Analysis to Identify Human-Machine Interface Design Challenges Unique to Uninhabited Aircraft Systems," Proceedings of the Human Factors and Ergonomics Society Annual Meeting, Vol. 56, No. 1, 2012, pp. 418-422.

[3] Pelham, J.G., Fan, I., Jennions, I., "Dual Use IVHM for UAS Health Management (SAE Technical Paper 2013-01-2202)," 2013,

[4] Healing, R.F., "Flight operations quality assurance (FOQA-HOMP)," 65th Annual Forum Proceedings - AHS International, Vol. 3, 2009, pp. 2167-2179.

[5] Holtom, M., "Properly managed FOQA programme represents an important safety tool for airlines." ICAO Journal, Vol. 55, No. 1, 2000, pp. 7-26.

[6] Newcome, L.R., "Unmanned aviation: a brief history of unmanned aerial vehicles," Pen and Sword, 2005,

[7] Whittle, R., "Predator's big safari," Arlington, Virginia: Mitchell Institute for Airpower Study.Url: Http://Www.Afa.Org/Mitchell/Reports/MP7_Predator_0811.Pdf.Accessed, Vol. 14, 2011, 
[8] Howard, M., "Air Force Safety Center RPA Branch," CNS / ATM 2011 Briefings, Vol. 1, Armed Forces Communications and Electronics Association, Boston, 2011,

[9] Osinga, F.P., "Science, strategy and war: The strategic theory of John Boyd," Routledge, 2007,

[10] Rasmussen, J., "Skills, rules, and knowledge; signals, signs, and symbols, and other distinctions in human performance models," Systems, Man and Cybernetics, IEEE Transactions On, Vol. SMC-13, No. 3, 1983, pp. $257-$ 266.

[11] Boyd, J.R., "A discourse on winning and losing," 1987,

[12] Beale, J.K.,II, and Hess, A., "Experiences with A-7E and the AV-8B engine monitoring systems: the good and the ugly," Aerospace Conference Proceedings, 2000 IEEE, Vol. 6, 2000, pp. 221-227 vol.6.

[13] ENDSLEY, M., and BOLSTAD, C., "Human capabilities and limitations in situation awareness," AGARD, Combat Automation for Airborne Weapon Systems: Man/Machine Interface Trends and Technologies 10 P(SEE N 93-28850 11-54), 1993,

[14] Onken, R., and Walsdorf, A., "Assistant systems for aircraft guidance: Cognitive man-machine cooperation," Aerospace Science and Technology, Vol. 5, No. 8, 2001, pp. 511-520.

[15] Billings, C., "Human-Centered Aviation Automation: Principles and Guidelines," NASA, NASA-TM-110381, Ames Research Center, Moffett Field, CA 94035 United States, 1996.

[16] Aamodt, A., and Plaza, E., "Case-based reasoning: Foundational issues, methodological variations, and system approaches," AI Communications, Vol. 7, No. 1, 1994, pp. 39-59.

[17] Letorneau, S., Famili, F., and Matwin, S., "Data mining for prediction of aircraft component replacement." IEEE Intelligent Systems Jr., Special Issue on Data Mining, 1999, pp. 59-66.

[18] Sarter, N.b., and Woods, D.D., "Cognitive engineering in aerospace application : pilot interaction with cockpit automation," NASA, NASA/CR-177617, Ames Research Center, Moffett Field, California, USA, 1993.

[19] Baxter, G., Besnard, D., and Riley, D., "Cognitive mismatches in the cockpit: Will they ever be a thing of the past?" Applied Ergonomics, Vol. 38, No. 4, 2007, pp. 417-423.

[20] Forrest, S., Perelson, A.S., Allen, L., "Self-nonself discrimination in a computer," Research in Security and Privacy, 1994. Proceedings., 1994 IEEE Computer Society Symposium on, Ieee, 1994, pp. 202-212.

[21] Hall, D.L., Hansen, R.J., and Lang, D.C., "The negative information problem in mechanical diagnostics," Journal of Engineering for Gas Turbines and Power, Vol. 119, No. 2, 1997, pp. 370-377.

[22] Cohen, I.R., "Tending Adam's Garden: evolving the cognitive immune self," Academic Press, 2000,

[23] Hofmeyr, and Forrest, "Architecture for an Artificial Immune System," Evolutionary Computation, Vol. 8, No. 4, 2000, pp. 443-473.

[24] Lee, J., Ghaffari, M., and Elmeligy, S., "Self-maintenance and engineering immune systems: towards smarter machines and manufacturing systems," Annual Reviews in Control, Vol. 35, No. 1, 2011, pp. 111-122. 
[25] Heaton, A., Fan, I., Lawson, C.P., "A Business Case for IVHM on Commercial UAS," UAS Operations: Dependable, Effective and Efficent? Royal Aeronautical Society, Royal Aeronautical Society, 4 Hamilton Place, London, 2012,

[26] Hunt, J.E., Cooke, D.E., and Holstein, H., "Case memory and retrieval based on the immune system," CaseBased Reasoning Research and Development, Springer, 1995, pp. 205-216.

[27] Dasgupta, D., and Nino, F., "Immunological computation: theory and applications," CRC Press, 2008,

[28] Bersini, H., Carneiro, J., Stibor, T., "On the Use of Hyperspheres in Artificial Immune Systems as Antibody Recognition Regions," Springer Berlin Heidelberg, 2006, pp. 215-228.

[29] Bradley, A.P., "The use of the area under the ROC curve in the evaluation of machine learning algorithms," Pattern Recognition, Vol. 30, No. 7, 1997, pp. 1145-1159.

[30] Flemming, J., "Utilizing FOQA (Flight Operations Quality Assurance) in training," 57th Annual Corporate Aviation Safety Seminar 2012, CASS 2012, 2012, pp. 52-60.

[31] Finnegan, M., "Boeing 787s to create half a terabyte of data per flight, says Virgin Atlantic ComputerworldUK.com," Computerworld UK, 2013,

[32] Atkinson, M.P., van Hemert, J.I., Han, L., "A distributed architecture for data mining and integration," Proceedings of the second international workshop on Data-aware distributed computing, ACM, 2009, pp. 11-20.

[33] Dean, J., and Ghemawat, S., "MapReduce: simplified data processing on large clusters," Communications of the ACM, Vol. 51, No. 1, 2008, pp. 107-113.

[34] Puttini, L., "IVHM Development and the Big Data Paradigm," SAE, 2013,

[35] Higginbotham, S., "How Formula 1 turns 3D printing, big data and crappy bandwidth into sport," 2013,

[36] Hundley, J., "Civil UAV Type Certification: DoD Mishap Analysis 2000-2009 and FAA Certification Roadmap," 2012,

[37] Saban, D., "Wake Vortex Modelling and Simulation for Air Vehicles in Close Formation Flight," 2010,

[38] Andrisani, D., "Flat Earth Simulation Software for MATLAB 5 or 6 with SIMULINK," 2010,

[39] Al Azzawi, D., "Aircraft Abnormal Conditions Detection, Identification, and Evaluation Using Innate and Adaptive Immune Systems Interaction," 2014, pp. 144.

[40] Moncayo, H., Perhinschi, M.G., and Davis, J., "Aircraft failure detection and identification using an immunological hierarchical multiself strategy," Journal of Guidance, Control, and Dynamics, Vol. 33, No. 4, 2010, pp. 1105-1114.

[41] Sandve, G.K., Nekrutenko, A., Taylor, J., " Ten Simple Rules for Reproducible Computational Research," PLoS Comput Biol, Vol. 9, No. 10, 2013,

[42] Scandura Jr., P.A., "Integrated vehicle health management as a system engineering discipline," 24th DASC: 24th Digital Avionics Systems Conference, Vol. 2, 2005, pp. 7.D.1-1. 
[43] Aaseng, G.B., "Blueprint for an integrated vehicle health management system," Digital Avionics Systems, 2001. DASC. 20th Conference, Vol. 1, 2001, pp. 3C1/1-3C1/11 vol.1. 
$2015-12-31$

\section{Application of an AIS to the problem of through life health management of remotely piloted aircraft}

Pelham, Jonathan G.

American Institute of Aeronautics and Astronautics

Pelham, J., Fan, I.-S., Jennions, I.K., McFeat, J., Application of an AIS to the problem of through life health management of remotely piloted aircraft, AIAA Infotech @ Aerospace.

Kissimmee, Florida, 2015

http://dx.doi.org/10.2514/6.2015-1797

Downloaded from Cranfield Library Services E-Repository 